\title{
Management of obese people: the others barriers
}

\section{Editorial}

Obesity is considered a multifactorial disease, with the participation of genetic, epigenetic, behavioural, socio-cultural and environmental factors, ${ }^{1}$ that produce an imbalance between expenditure and energy intake, during long time. It is often unrecognized that although obesity was included in the classic listing of diseases in 1948, the doctors dismissed it for decades., ${ }^{2,3}$ This was probably related to the fact that obesity was considered a reversible problem, that can be solved if people eat less and increase their physical activity, this misunderstanding persists today, and is at least in part responsible for the increase in incidence and prevalence of obese people that reaches a magnitude that is considered an epidemic around the world.

Other factors such as less sleep, cessation of smoking, weight gain associated with some medications, older parental age at birth, and endocrine disruptors are factors recognized as contributors to such a situation. ${ }^{4}$ The aforementioned factors explain the increase in the prevalence of obese people throughout the world, but they have little, or no value when we are in front of a patient, as discussed below.

In general, the treatment of people with obesity is based on a change in lifestyle that includes changes in their eating pattern and increase in physical activity, this seems easy to understand presents many problems when it comes to doing it. ${ }^{5}$ The majority of patients leave the treatment after a few weeks or months. The lack of adherence to the different regimes may due to:

a. Not understand very well the bases of such regimens,

b. Not lose the weight they expected,

c. The patient understands that, as occur with other diseases, the health professional has to make more effort than merely saying the patient what should change in their lives.

d. Patients realize that for the regime to be effective, the patient has to make a great effort, for a long time, and that it is not worth depriving himself of things that he likes.

e. In general, patients are not told that in order for the regime to be successful, it has to change its behaviour for life;

f. Commercial diets emphasize only short-term weight loss.

g. The widespread belief that if you lose weight it is a success even if you later recover the weight, when in fact it is a failure.

h. Frequently obese patients have behavioural alterations that condition the development of obesity and the difficulty in their treatment, especially if they are not diagnosed. ${ }^{6,7}$ These and other similar reasons are the main barriers to the success of the different programs to lose weight to lose and not get it back.

The key question is why that happens, the answer is not unique either. In almost all countries around the world, health care is characterized by a provider-centred model, ${ }^{8}$ rather than peoplecentred model, with an emphasis on the diagnosis and treatment of diseases that obviously gives little importance to the individual characteristics of each patient, basic to their management. Lack of qualified and experienced health personnel to treat such complex patients. Surprisingly, many of the so-called obesity experts are not

\author{
Volume 6 Issue 3 - 2018
}

\section{RicardoV Garcia Mayor}

South Galicia Biomedical Research Institute and Endocrine Unit, HM Hospital of Vigo, Spain

Correspondence: Ricardo V Garcia Mayor, South Galicia Health Research Institute, University Hospital of Vigo, Technic Block, 2nd floor, 34I Clara Campoamor Street, 36312 Vigo, Spain, Tel + 34/630/963 866,

Email ricardo.garcia.mayor@sergas.es

Received: May 25, 2018| Published: June 05, 2018

clinicians. Unfortunately, very often the health managers to lower costs, and with the support of specialists, who feel unable to treat these difficult patients, refer them to the primary care or nursing units.

\section{Conclusion}

The improvement in the management, not of obesity, but of patients with obesity begins with the qualification of the professionals who have to treat them, with sufficient experience; it is not enough to follow valid protocols for all.

\section{Acknowledgments}

None.

\section{Conflict of interest}

The Author declares there no conflict of interest.

\section{References}

1. Bray GA, Frübeck G, Ryan DH, Wilding JP. Management of obesity. Lancet. 2016;387(10031):1947-1956.

2. James WT. Research on obesity. A report of the DHSS/MRC group. London: HMSO; 1976.

3. Coll JR. A report of the Royal College of Physicians. Physicians London. 1983;17:5-65.

4. Garcia-Mayor RV, Larranaga A, Docet MF, et al. Endocrine disruptors and obesity: Obesogens. Endocrinologia y Nutricion. 2012;59(4):261267.

5. Cordido F, Garcia-Mayor RV, Larranaga A. Obesity, Adipose Tissue, Inflammation and Update on Obesity Management. Obes Control Ther. 2014;1:1-8.

6. Larranaga A, Garcia-Mayor RV. High frequency of non-specified eating disorders in obese persons. Nutricion Hospitalaria. 2009;24:661-666.

7. Docet MF, Larranaga A, Perez Mendez LF, et al. Attention deficit hyperactivity disorder increases the risk for having abnormal eating behaviours in obese adults. Eat Weight Disord. 2012;17(2):e132-136.

8. Laine C, Davidoff F. Patient-centered medicine. A profesional evolution. JAMA. 1996;257(2):152-156. 